\title{
Gradhiva
}

GRADHI

Revue d'anthropologie et d'histoire des arts

$23 \mid 2016$

Collections mixtes

\section{Leiris, singulier pluriel}

\section{Éléonore Devevey}

\section{(2) OpenEdition}

\section{Journals}

Édition électronique

URL : http://journals.openedition.org/gradhiva/3198

DOI : 10.4000/gradhiva.3198

ISSN : 1760-849X

\section{Éditeur}

Musée du quai Branly Jacques Chirac

\section{Édition imprimée}

Date de publication : 25 mai 2016

Pagination : 214-227

ISBN : 978-2-35744-093-7

ISSN : 0764-8928

Référence électronique

Éléonore Devevey, « Leiris, singulier pluriel », Gradhiva [En ligne], 23 | 2016, mis en ligne le 25 mai 2016, consulté le 23 septembre 2020. URL : http://journals.openedition.org/gradhiva/3198 ; DOI : https:// doi.org/10.4000/gradhiva.3198 


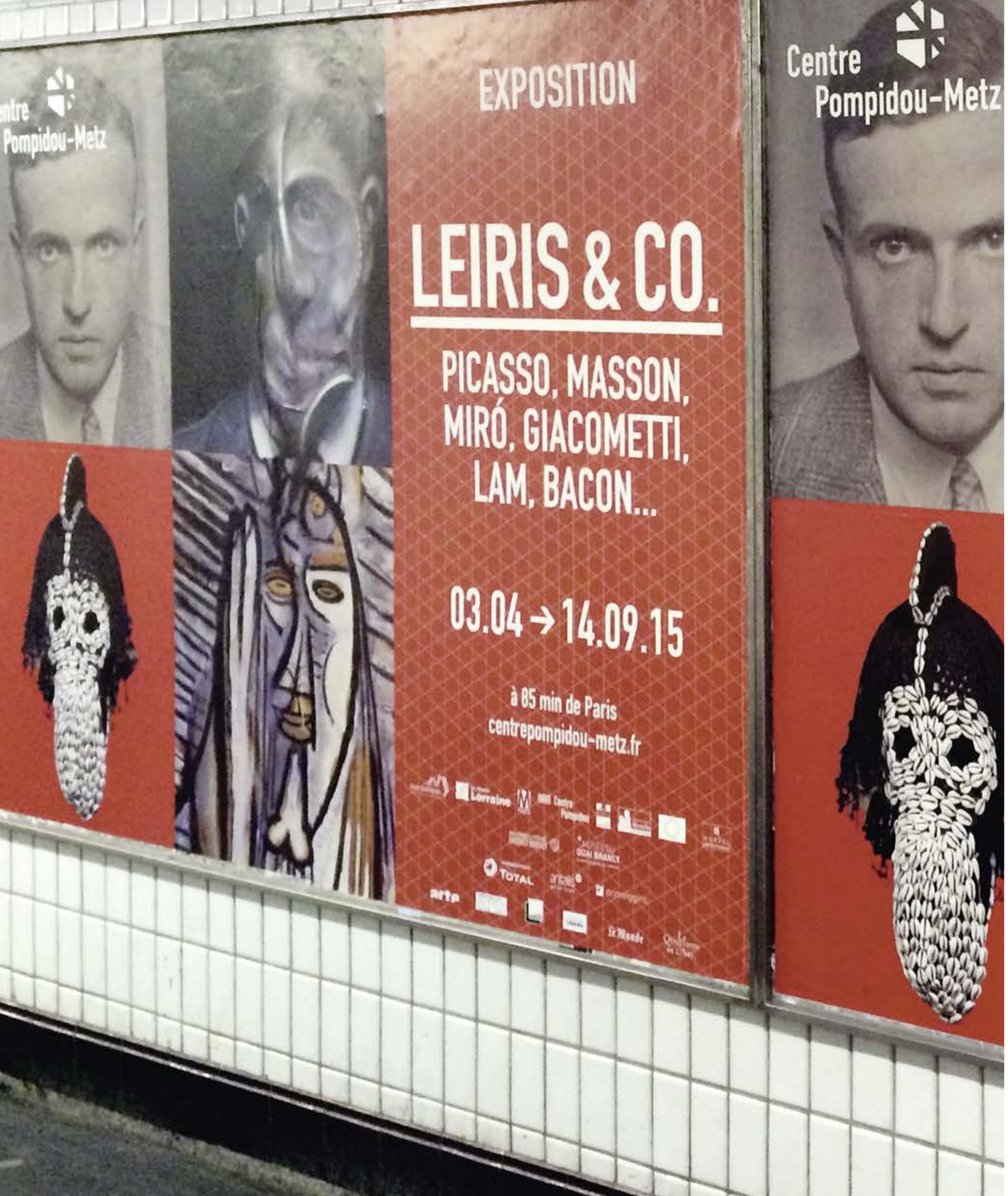

Centre

Pomnidiol- NEtz

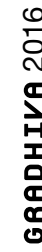

23 


\section{Leiris, singulier pluriel}

\section{par Éléonore Devevey}

Agnès de La Beaumelle, Marie-Laure Bernadac et Denis Hollier (dir.), Leiris \& Co. Picasso, Masson, Miró, Giacometti, Lam, Bacon...

Paris, Gallimard-Centre Pompidou-Metz, 2015, 400 p.

De même que «la science» pourrait se réduire, suivant Monsieur Teste, à «des savants et des moments de savants» (Valéry 1978: 101), la vie artistique, littéraire, intellectuelle se joue parfois dans un and co: co-writers, co-founders, co-workers, co-brothers même - comparses et collaborateurs, collègues et confidents, compagnes et comètes. Porter ainsi attention à des moments de vie partagés autant qu'aux produits de ces activités, s'attacher aux complicités et aux compagnonnages qui les portent a la vertu de saper l'unité idéale de la science comme des arts tout en leur redonnant leur densité existentielle.

Tel a été le parti de l'exposition Leiris \& Co et du catalogue qui en procède, tous deux dirigés par Agnès de La Beaumelle, Marie-Laure Bernadac et Denis Hollier, avec le concours de Jean Jamin, conseiller scientifique: montrer Michel Leiris entouré, Leiris singulier pluriel. Pluriel, parce que son œuvre prend forme à travers un réseau de rencontres et d'échanges; singulier, parce qu'elle reste celle d'un irréductible outsider, travaillé par le souci de soi. Pour aborder sa vie, sans doute la mieux documentée de toutes les vies d'ethnologues français - par les soins acharnés du diariste, de l'autoportraitiste, de l'autobiographe qu'il fut, par le travail non moins passionné de ses exégètes -, c'est l'adage convoqué par André Breton au seuil de Nadja qui définit ici la focale: «Dis-moi qui tu hantes, je te dirai qui tu es. " Soit, dans les termes de l'intéressé:

Mais qu'est-ce qu'un je - un je unique et isolé - sans un tu, sans un nous, sans un il gravitant autour de lui et fournissant matière à une hiérarchie dont il occupe le sommet, personne première et singulière? Arraché du je-tu-il, retranché du nous-vous-ils (sans parler du je-tuELLE) le je, dans d'aussi évidemment absurdes conditions, n'est plus guère qu'un enfant qui, voulant se compter tout seul, récite am-stramgram pic-et-pic-et-colégram bour-et-bour-et-ratatam mis-ta-gram et tourne indéfiniment en rond dans cette combinaison de paroles et de mouvements réduite à un vain bredouillement, puisqu'il n'y a pas là de compagnons à la fois opposés et associés à lui pour justifier un tel dénombrement et façonner, en dressant devant lui le môle d'autrui, les limites qui lui confèrent une existence en tant que corps séparé ${ }^{1}$ ? (Leiris 2003: 71)

C'est donc par le biais de ses contemporains, autant d'astres qui gravitent dans le système leirisien et en dessinent les cercles, qu'est ressaisi l'écrivain, ainsi soustrait au solipsisme de la comptine. Ce catalogue d'exposition, écrit à plusieurs mains, évoquant plusieurs vies, désigne à sa façon
1. Cité par Peter Szendy, p. 204. 
2. Nous ne pouvons bien sûr pas considérer ici chacune de ces contributions; nous indiquerons simplement, entre parenthèses, le nom des contributeurs que nous citons. Quand nous ne le précisons pas, c'est que nous empruntons à la chronologie qui court sur l'ensemble du volume.

3. Lettre à André Castel, 19 avril 1944, citée p. 206.

\section{ci-contre}

fig. 1

Couverture du catalogue de l'exposition Leiris \& Co...,

Paris-Metz, Gallimard/Centre

Pompidou-Metz, 2015 un idéal de la biographie: un récit fragmentaire qui, ayant pour amorce les traces matérielles d'affinités électives, prendrait au sérieux le parti de s'attacher à toutes les vies connexes à celle du biographié, ainsi ramenée à cette constellation.

\section{Leiris, brisées reliées}

La mise au point reste, en fait, sur la figure centrale, mais l'ensemble permet néanmoins d'appréhender simultanément figure et fond. Les courts textes des trois commissaires de l'exposition, de Jamin et des quarante-cinq contributeurs $^{2}$ (dont six plasticiens, témoignant de l'empreinte d'un «Leiris fantôme" sur leur travail), centrés sur une relation privilégiée ou un fait circonscrit, progressent par diffraction, ténus chevauchements (qui ont l'avantage d'indiquer au profane les faits saillants, également remis en musique par la chronologie), avancées dans le temps. Ils proposent des portraits à deux têtes, en "pas de deux", à un moment $t$ ou sur le plus long terme, éclairent la participation de Leiris à un collectif ou à un événement, les circonstances d'une rencontre, la naissance d'une passion, l'invention ou le creusement d'une forme.

C'est d'abord le(s) Leiris de Brisées et de Zébrage, ou des Écrits sur l'art, que le volume met à l'honneur; Leiris lui-même auteur, préfacier ou contributeur de catalogues d'exposition: celles d'André Masson, Pablo Picasso, Alberto Giacometti, Francis Bacon, mais aussi Joan Miró, Élie Lascaux, Wifredo Lam... - toujours des amis, Leiris n'ayant écrit «que sur des artistes avec lesquels il avait des relations personnelles, voire métafamiliales» (p. 210). L'iconographie, somptueuse, donne bien sûr accès à la réciproque: Leiris, face et profils, vu par ses contemporains. Sur le rabat du volume, un portrait photographique par Man Ray, frontal; sur son verso, un pastel par Picasso, profil droit; en dessous, en page de couverture, une huile de Bacon, profil gauche - datés respectivement de 1930, 1963 et 1976. Donnée cardinale des voies de la création, celle, aussi, dont l'historicité est la plus labile, les amitiés d'atelier marquent la vie de Leiris de leurs scansions - «aux moments critiques de son évolution, note Étienne-Alain Hubert, on le sent tenté de découvrir dans les autres les voies qui lui permettront de se trouver en tant qu'homme et, virtuellement, en tant qu'écrivain " (p. 29) -, aussi bien qu'elles proposent une traversée des modes de sociabilité intellectuelle au $x x^{e}$ siècle. Leiris s'est trouvé pris tour à tour dans de petits collectifs, aux forces centripètes et centrifuges, qui génèrent quelques relations privilégiées: le 45 , rue Blomet, l'atelier de Masson, qui l'introduit à la tribu Kahnweiler, le surréalisme, puis sa dissidence, les "missions", puis les revues, les collèges de sociologie, puis de 'pataphysique... Dans la «sorte de petite bande d'inséparables (dont je suis)" formée en 1944 «à la faveur des circonstances, dans la plus pure amitié ${ }^{3}$ " (et qui compte Jean-Paul Sartre, Simone de Beauvoir, Albert Camus ou Raymond Queneau), c'est l'assurance de liens noués dans une «maudite époque» qui lui importe: refuge devant l'adversité, ces complicités sont aussi la condition de l'humour, la connivence revigorant les convictions en même temps qu'elle favorise l'autodérision - ce dont témoigne la lecture collective, chez les Leiris, de la pièce de Picasso, Le Désir attrapé par la queue, en mars 1944. Si, par ailleurs, le sous-titre de l'exposition oubliait les figures féminines, l'ouvrage pourtant leur fait place, quoique en mode mineur: des contributions sont consacrées aux figures de Zette et de Laure; 

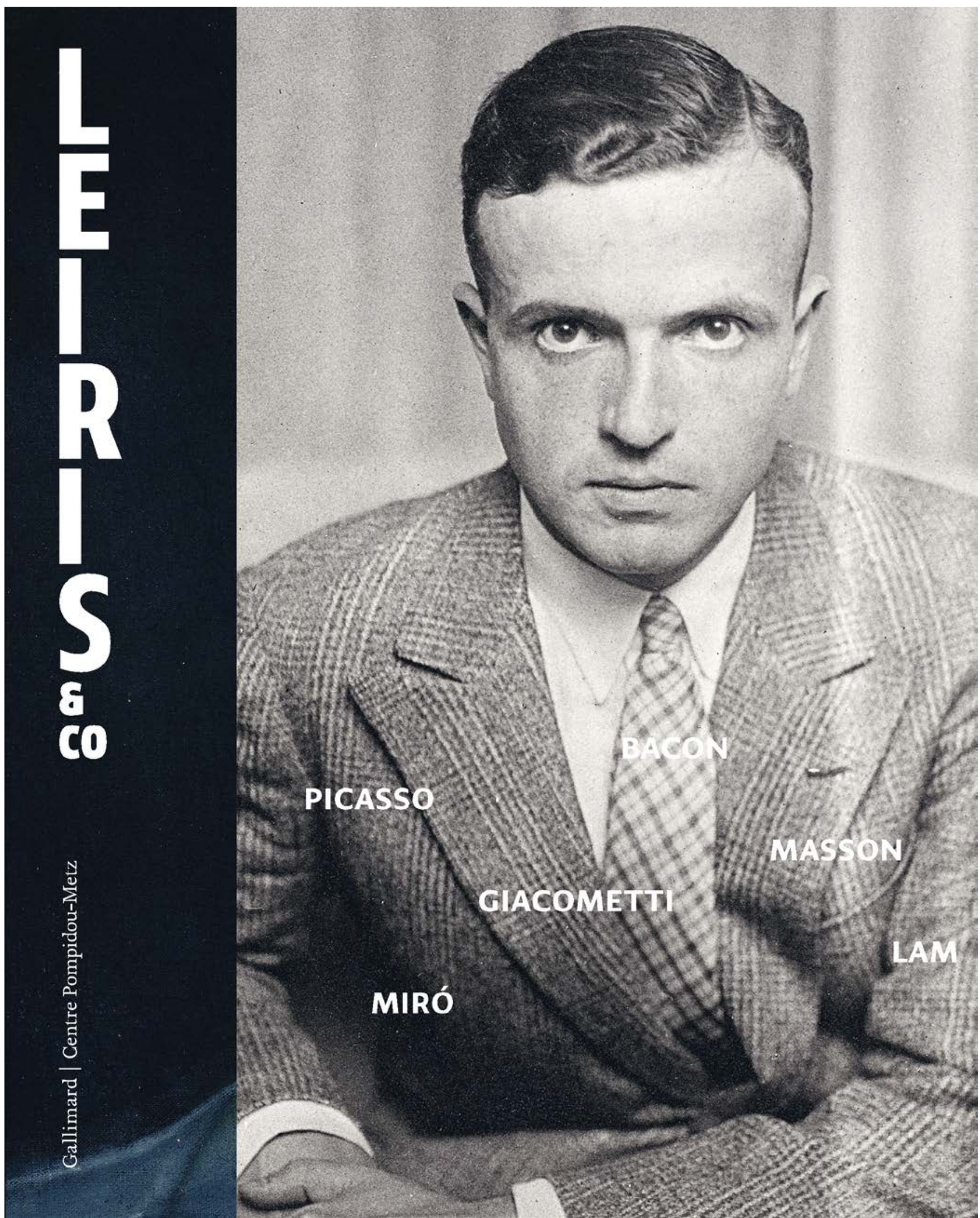


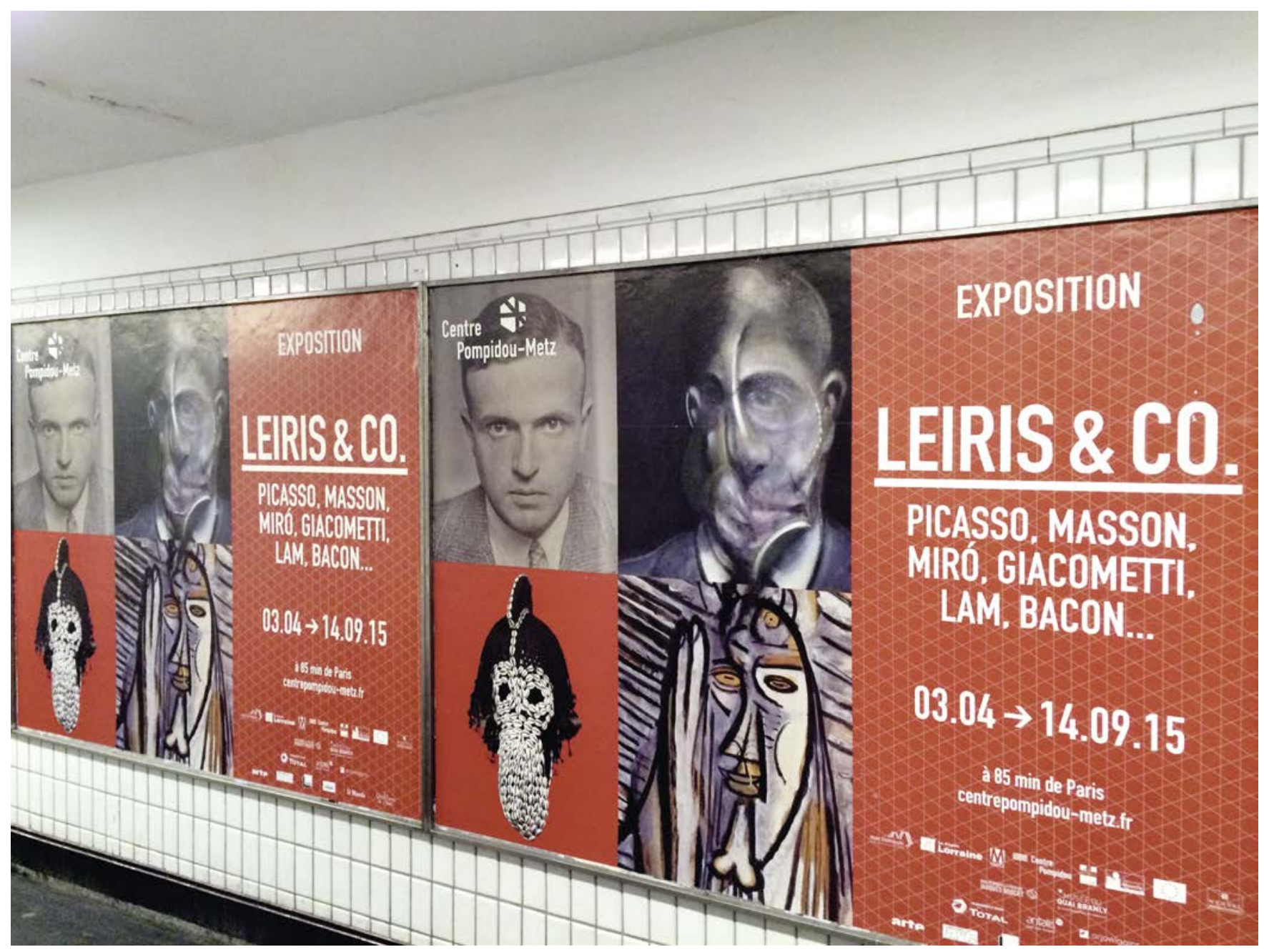


on croise, de façon plus fugace, Emawayish ou Khadidja; allusivement, Hélène Gordon, Pauline Roux, Germaine Rouvre ou Sonia Orwell - sans omettre la part féminine propre à Leiris, qui, selon Gérard Cogez, aurait pu dire: «Olympia, c'est moi. »

Pluriel, Leiris l'est donc aussi par les voies du dedans: ce que laissent voir, outre ses multiples portraits et ses avatars fictionnels, chez Max Jacob ou Marcel Jouhandeau, le jeu des pseudonymes ou des doubles pratiqué par Leiris lui-même - Leiriset Masson, donné pour l'auteur de Simulacre (poèmes de Leiris illustrés par Masson), Damoclès Siriel (dans Aurora), ou la figure d'Axel Heyst, empruntée à Joseph Conrad ${ }^{4}$-, ou encore sa fascination pour la possibilité d'user d'un «vestiaire de personnalités " (Leiris 1996: 942) et pour les phénomènes de possession - qui sont la chance, précisément, de se laisser au vestiaire. Leiris fasciné, mais sans illusions, par ce que Charles Baudelaire concevait comme l'attribut du poète, «cet incomparable privilège, qu'il peut à sa guise être lui-même et autrui ». On retrouve, dans ces affinités électives comme dans ces velléités de métamorphose, ce qu'on pourrait appeler le mécanisme de la double détente: celui qui consiste à singulariser pour dépasser le singulier - se définir pour mieux se dissoudre, s'indéfinir pour ne pas s'arrêter. Ce mécanisme, les compagnonnages le relancent puisqu'ils invitent Leiris à spécifier ce qui chez d'autres l'appelle ou le déstabilise, mais aussi à reconnaître en eux ce qui l'anime également.

\section{Leiris: initié au sérieux de la science}

Centré sur les arts, le volume ne néglige pourtant pas la «vie savante $\mathbf{5}^{\mathbf{}}$ » de Leiris, qui a elle aussi son iconographie, permet elle aussi de jouer avec les masques et d'essayer la persona scientifique. Elle s'agence autour des deux foyers que sont la mission Dakar-Djibouti, devenue lieu de mémoire de l'ethnologie française, et le tournant de l'engagement, dont l'articulation est finement restituée.

La «mission», qui s'est jouée pour Leiris par l'entremise de l'ondoyant Georges Henri Rivière et du martial Marcel Griaule, prend à Sanga, où il est séduit par la langue rituelle des Dogons, pour s'intensifier à Gondar, dans le voisinage des possédées. Si elle nourrit encore sa fascination pour le secret et l'antérieur, elle a aussi une indéniable valeur initiatique, sur les plans professionnel et personnel. Dans le sillage du travail réalisé pour la réédition en Pléiade de L'Afrique fantôme et de L'Âge d'homme (2014), Hollier reprend les fils des chantiers qu'elle noue, au cours de ces décisives années 1930, moment de tectonique des régimes d'écriture entre lesquels se révèle pourtant la même hantise de la disparition. Au musée de l'Homme, dans lequel, Afrique traversée et diplômes en poche, Leiris a trouvé ses quartiers, son attitude, quand s'organisent les premiers groupes de résistance, est à la fois de refus et de repli. La guerre aura été vécue à contretemps: commencée en Beni Ounif, où il est mobilisé comme chimiste de fortune, elle s'achève sur un déboire à Dakar, au terme de la mission Lucas (février-mai 1945, en Côte d'Ivoire et en Gold Coast) qui «permet à l'Afrique jusqu'alors "fantôme " [...] de prendre réellement corps » (Marianne Lemaire, p. 212) et engage à repenser la «fraternité » à l'heure des indépendances. Ce nouvel usage de la science et de soi comme la distribution des régimes d'écriture qui en ressort sont signifiés dans le prière d'insérer d'Aurora (écrit en 1927, publié en 1946),
4. Ce personnage figure dans le récit esquissé lors de la halte à Agordat, à la fin de L'Afrique fantôme; voir Denis Hollier, p. 142.

5. Signalons la publication à venir, dans la Revue d'histoire des sciences humaines, d'un article exposant la mise en œuvre de cette notion par des anthropologues - Nicolas Adell, "La vie savante comme objet historique et anthropologique "-, qui est l'objet du programme VISA, «Vie savante et anthropologie».

\section{ci-contre}

fig. 2

Campagne publicitaire dans le métro parisien (ligne 7 , station Jussieu), annonçant l'exposition Leiris \& Co... au Centre Pompidou-Metz, avril 2015. D.R. 
fig. 3

Installation de la vitrine consacrée à L'Afrique fantôme, exposition Leiris \& Co..., Centre PompidouMetz, 27 mars 2015. D.R.
Documents Bleus Notro temp! MICHEL LEIRIS

\section{LAfrique} fantôme

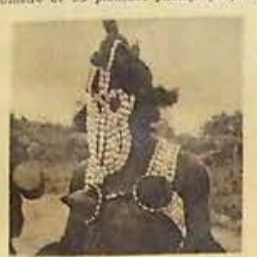

Librairie בGallimard

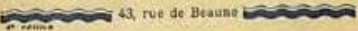
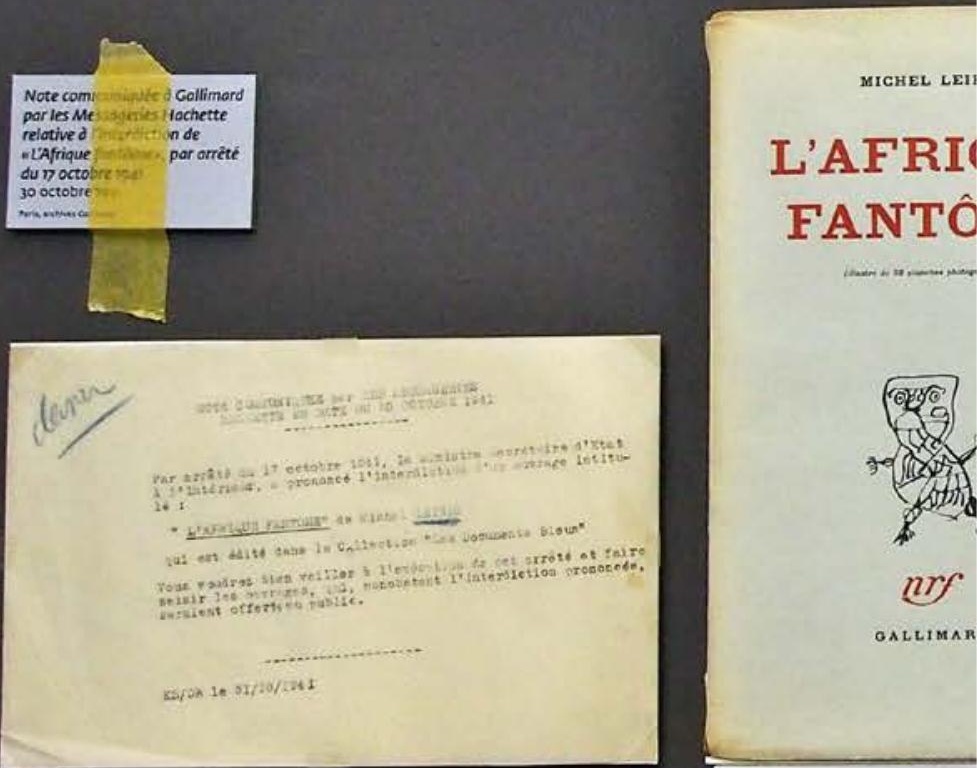

ILIMAR

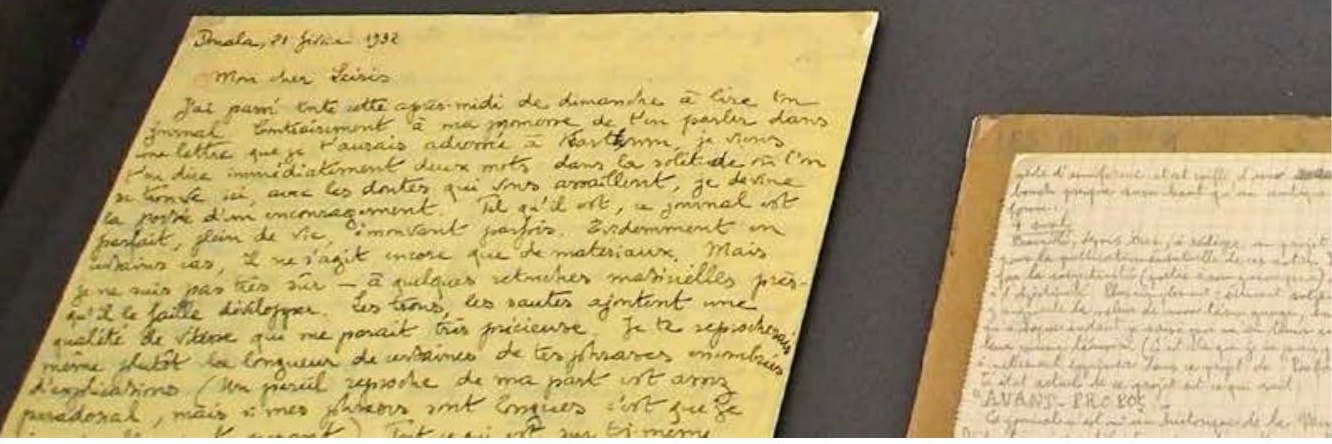


6. Cité par Jean Jamin, p. 257.

7. La "Notice» de L'Afrique fantôme, dans l'édition de la Pléiade, éclaire de son côté les rapports entre Michel Leiris et Marcel Griaule (Leiris 2014: 1016-1018). qui indique que «le temps de Michel Leiris est aujourd'hui partagé entre son activité littéraire et son appartenance professionnelle à une science qu'il envisage, maintenant, moins sous l'angle de la connaissance que pour la contribution qu'elle peut apporter à l'affranchissement des hommes d'outre-mer » (cité p. 214).

La genèse de sa pensée des rapports entre ethnographie et colonialisme est ici reprise par Jamin: si L'Afrique fantôme a été l'écran de projection de toutes les «crises » ultérieures de l'ethnologie, elle est d'abord un journal intime dans lequel «il se livre», «énonce mais ne dénonce pas» (p. 254-255); ce n'est que tardivement, après la rencontre avec Aimé Césaire et le premier séjour aux Antilles en 1948, que sa conscience politique alors sommaire prend véritablement consistance. La vocation de l'ethnographe à être l'«avocat» des sociétés qu'il étudie, définie dans l'article de 1949, "L'ethnographe devant le colonialisme», qui deviendra le levier de l'infléchissement de son rôle après Bandung, est réaffirmée en 1961 pour justifier sa signature du Manifeste des 121 : il est «leur avocat désigné, celui qui plus que quiconque doit s'attacher à faire admettre leurs droits, sans excepter le droit de lutter à leur tour pour se constituer en nation ${ }^{6}$ » (Leiris 1992a: 912). Cette orientation ne s'impose cependant pas sans éclipse: c'est ce dont témoigne un manquement à la déontologie de la publication ethnologique, «reste d'inconscience coloniale» qui lui fait oublier qu'«il risque d'être lu par ceux dont il parle» (Vincent Debaene, p. 228), et ce malgré sa participation à Présence africaine et, plus tard, au premier Festival mondial des arts nègres. On le retrouve par ailleurs enthousiaste à l'égard de la révolution cubaine et de Mai 68, lorsqu'il "participe avec Robert Jaulin et Jean Malaurie à un enseignement "critique" et "polémique" des théories ethnologiques initié par Jaulin parallèlement aux cours officiels de la Sorbonne» (p. 324), ou concerné par les conditions de vie de réfugiés maliens. Ses engagements conditionnent les inflexions d'une culture contestataire qui passe, idéologiquement, de l'anticolonialisme à l'anti-impérialisme, mais accompagnent aussi les infléchissements qui affectent le paysage intellectuel, dans lequel les écrivains cèdent peu à peu le pas aux universitaires, aux «intellectuels spécifiques».

II y a sans doute d'autres «co-» dont, dans ce domaine, on aurait pu espérer le portrait croisé (mais dont le compagnonnage est sans doute moins visuel): Abba Jérôme (son interprète à Gondar, que l'on retrouve invité au Collège de 'Pataphysique), Alfred Métraux (dont les carnets alimentent pourtant la chronologie des années 1951-1952), Georges Balandier (dont Leiris fut le mentor), ou Jamin lui-même, qui fonde avec lui (et Jean Copans) la collection "Les hommes et leurs signes", aux éditions du Sycomore, puis la revue Gradhiva, et qui devient son exécuteur testamentaire puis son éditeur ${ }^{7}$. Les contributions peuvent également faire espérer un volume qui, à l'instar de Miroir de l'Afrique, rassemblerait tous les écrits de Leiris autour des Antilles (ses carnets de route sont encore inédits). Leiris a en somme, vis-à-vis de la science comme de la littérature, un statut double: celui d'initié et celui de franc-tireur. C'est sans doute le scepticisme qu'il nourrit également à leur égard qui favorise la recherche de "grands astreignants " et d'«alliés substantiels », leur compagnie ayant la vertu d'alléger les affres que suscite leur exercice. Si la vie de Leiris semble aussi intensément traversée par d'autres que rétive à leur faire véritablement place, c'est que leur contact 
est pour lui un aiguillon et un indicateur du réel, que le doute parfois dissout: ils l'authentifient, en révèlent à la fois les risques et la valeur.

\section{Leiris - vie prise en main par l'écrit?}

Le catalogue, davantage que l'exposition, rend compte du travail d'écriture de Leiris: tentative, aussi passionnée que vouée à l'échec, de prendre sa vie en main par l'écrit. C'est ce qu'atteste l'attention portée à la topographie cloisonnée de l'écriture (analysée par Jamin) ou sa matérialité (la pratique des fiches, ici abordée par Michael Sheringham), façons maniaques d'ordonner son existence. L'espoir que l'œuvre opère, et d'abord sur son créateur, traverse les formes qu'il invente, de la glose, qui se lit «comme un programme lyrique qui surdétermine tout à la fois la vie et l'écriture de l'auteur» (Marie-Paule Berranger, p. 62), au «truc » de La Règle du jeu, «un livre qui ne serait qu'un moyen dont la vie serait la fin» (Denis Hollier, p. 217), même si moyen et fin, Leiris s'en rend vite compte, vont bientôt s'inverser - et Hollier se demande d'ailleurs si l'écrivain a pu croire à son mythe. On retrouve aussi cet effort dans un trait stylistique, le «donc» en tête de phrase, façon de se tenir debout que Marielle Macé réinscrit dans « une histoire longue du stoïcisme, des duretés du rapport à soi et des recherches que l'on qualifiera de "dandysme moral” » (p. 169); dans l'idée qu'on peut “[vaincre son] destin d'homme à l'aide des mots 8 " (Leiris 2014: 880); dans le rapport à l'engagement - «beaucoup plus qu'à une "littérature engagée", note Leiris en 1945, je crois à une littérature qui m'engage " (Leiris 1992a: 421) -, qui apparaît alors comme une façon de s'obliger soi-même, d'exercer une pression sur sa vie à venir en étant tenu par le regard des autres. La gageure que représente ce désir de stylisation de soi est, avec le temps, prise avec plus de recul, et les objectifs revus à la baisse- «je est un autre, conclut-il dans un texte consacré à Picasso, mais nul ne peut arriver, grâce à son art, au miracle que serait l'authentique possession de soi ${ }^{9}$ " (Leiris 1992d: 92). L'écriture a alors une fonction d'apaisement - «Changer l'angoisse en mélancolie. Ainsi pourrais-je formuler ce qui est, peut-être, ma principale raison d'écrire ${ }^{10}$ » (Leiris 1992a: 738) - ou devient manière d'apprivoiser la mort lorsque Leiris renoue tardivement avec les gloses - «N'en pouvant mais, essayer du moins de me faire cadavre exquis 11 » (ibid.: 759 ).

Si la littérature reste la dominante de sa vie, Leiris voit sans doute aussi, dans l'écriture savante ou les pratiques artistiques, d'autres façons, toujours un peu désespérées, d'exercer une action sur soi, comme si l'œuvre, quelle qu'elle soit, procédait d'un volontarisme dont l'objet est l'existence, mais qui doit d'abord trouver la médiation d'une forme. Le «commun » des «co-» leirisiens pourrait ainsi être la disposition à chercher dans l'ouvrage la chance d'une transformation de soi, mais sans jamais y croire tout à fait. Ce qui non pas rassemble ces contemporains, mais tous, à un moment donné, les traverse, c'est sans doute une façon de se sentir reliés par le travail, ce dont témoigne une très belle définition donnée par Leiris après la mort de Giacometti des compagnons: «= camarades de travail ou, à tout le moins, camarades dont l'idée qu'ils sont au travail encourage ${ }^{\mathbf{1 2}}$ » (ibid.: 613). C'est la reconnaissance, les uns chez les autres, de cette exigence à la fois titanesque et dérisoire qui se joue entre Leiris et Picasso, depuis l'apostrophe du peintre à l'apprenti poète en 1924 - «Bonjour Leiris! Alors, vous travaillez?» - à l'observation de l'écrivain - «Ma vie se sera passée, pour
8. Cité par Gérard Wajcman, p. 284.

9. Cité par Marie-Laure Bernadac, p. 263.

10. Cité p. 349

11. Cité par Laurent Jenny, p. 360 .

12. Cité par Agnès de La Beaumelle, p. 90. 
13. Cité par Jean Frémon, p. 335 .

14. Titre de la préface écrite pour La Musique et la transe de Gilbert Rouget, repris dans Leiris 1992c : 201-212.

15. Titre d'un texte consacré à Fernand Léger, repris dans ibid. : 115. une large part, à essayer de justifier l'intérêt qu'il m'avait si gentiment témoigné» (Leiris 1992d: 83) -, ou encore dans la compagnie que Leiris trouve dans un autoportrait de Bacon - «Voisinage revigorant et appel au travail: un visage qui pèse tout son poids de chair et de peinture ${ }^{13}$ » (Leiris 1992a: 924). Leiris et ses contemporains partagent peut-être une façon de «se mettre au travail », expression à prendre littéralement et dans tous les sens: prendre date avec soi-même et s'engager dans le temps, se mettre soi-même à la question, accoucher d'un moi nouveau, travail de greffier, de tortionnaire ou de parturiente, qui suppose de «ne pas se contenter d'être ce que l'on est ${ }^{\mathbf{1 4}}$ ».

Quand l'exercice de la biographie intellectuelle a longtemps consisté à aller de la vie vers l'œuvre, celles de Leiris nous incitent résolument à prendre le courant à rebours pour aller des œuvres vers la vie. C'est dans cette perspective que se mesure «le savoir-vivre du créateur ${ }^{15}$ »: les œuvres sont pour lui l'espoir, toujours relancé, de s'inventer un contrat d'existence. Si on lit en suivant son ordre ce vaste portrait polyscopique et polyphonique, on peut avoir l'impression, comme dans un livre illustré dont les images devanceraient le texte, que le propos des contributions est légèrement en avance sur la chronologie: c'est le propre de l'écriture historienne, qui pour parler d'un fait même restreint doit cadrer plus large et porter plus loin que lui, mais c'est aussi l'effet du travail du créateur, qui s'essaie à préparer le terrain à la vie. Chez l'écrivain qui a «soif de l'impression qu'il ressent quand il est au travail et que cela marche à souhait», méthode pour «n'être plus sous la coupe de la mort» (Leiris 1989: n.p.), les œuvres ont une portée prospective, même - surtout - devant la fin. 


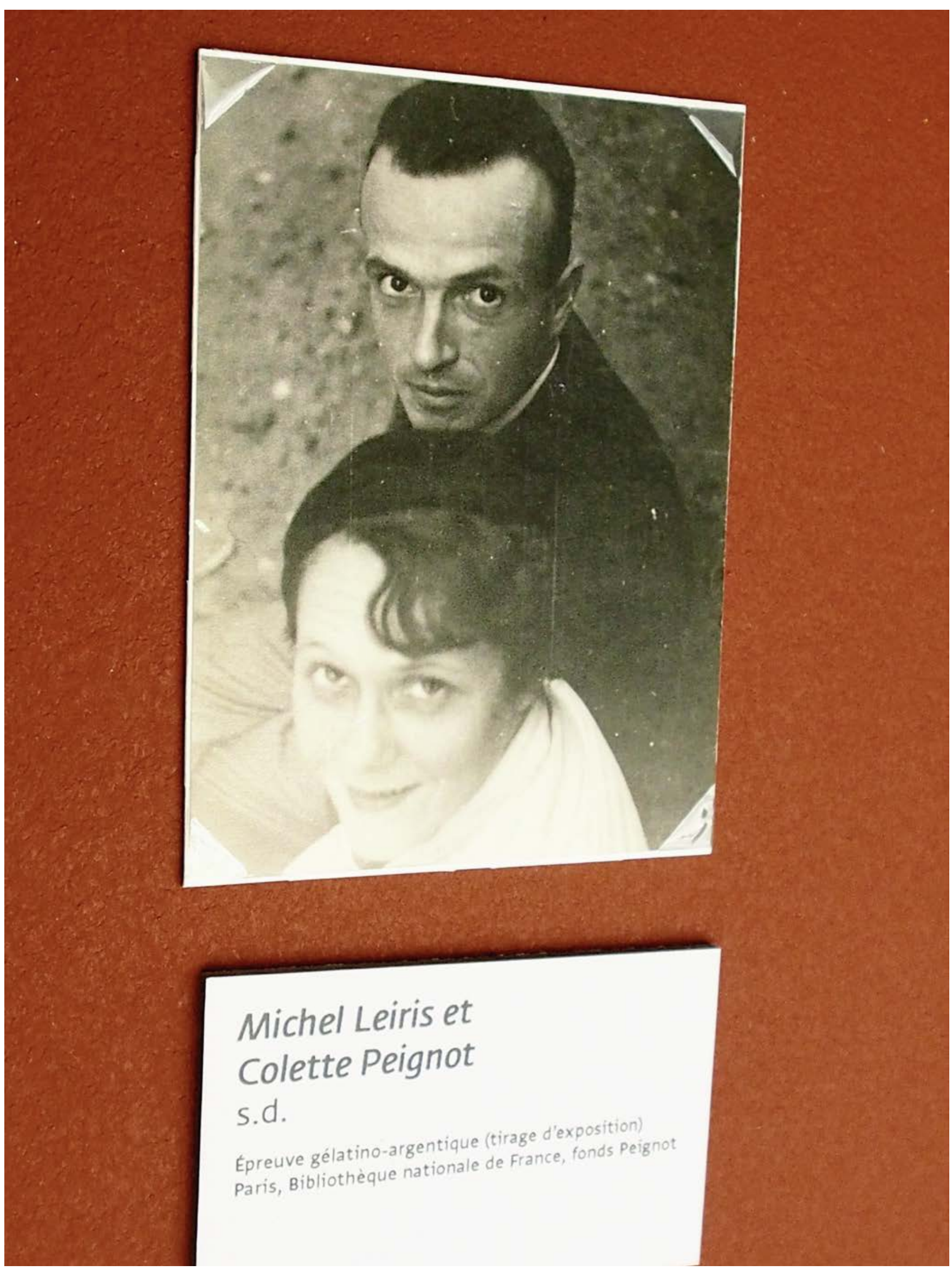




\section{Bibliographie}

\section{Leiris, Michel}

1989 Images de marque.

Cognac, Le Temps qu'il fait.

$1992 a$ Journal 1922-1989.

Paris, Gallimard.

1992b [1966] Brisées. Paris, Gallimard ("Folio essais").

1992c Zébrage. Paris,

Gallimard («Folio essais»).

1992d Un génie sans piédestal, et autres écrits sur Picasso.

Paris, Fourbis.

1996 Miroir de l'Afrique, édition de Jean Jamin. Paris, Gallimard ("Quarto").

2003 La Règle du jeu, édition dirigée par Denis Hollier.

Paris, Gallimard ("Bibliothèque de la Pléiade»).

2011 Écrits sur l'art, édition de Pierre Vilar. Paris, CNRS Éditions.

2014 L'Âge d'homme, précédé de L'Afrique fantôme, édition dirigée par Denis Hollier. Paris, Gallimard ("Bibliothèque de la Pléiade»).
Leiris, singulier pluriel.

Par Éléonore Devevey

\section{Valéry, Paul}

1978 Monsieur Teste. Paris,

Gallimard ("L'imaginaire»). page 214 et ci-contre

Campagne publicitaire dans le métro parisien (ligne 7 station Jussieu), annonçant l'exposition Leiris \& Co. au Centre Pompidou-Metz, avril 2015. D.R
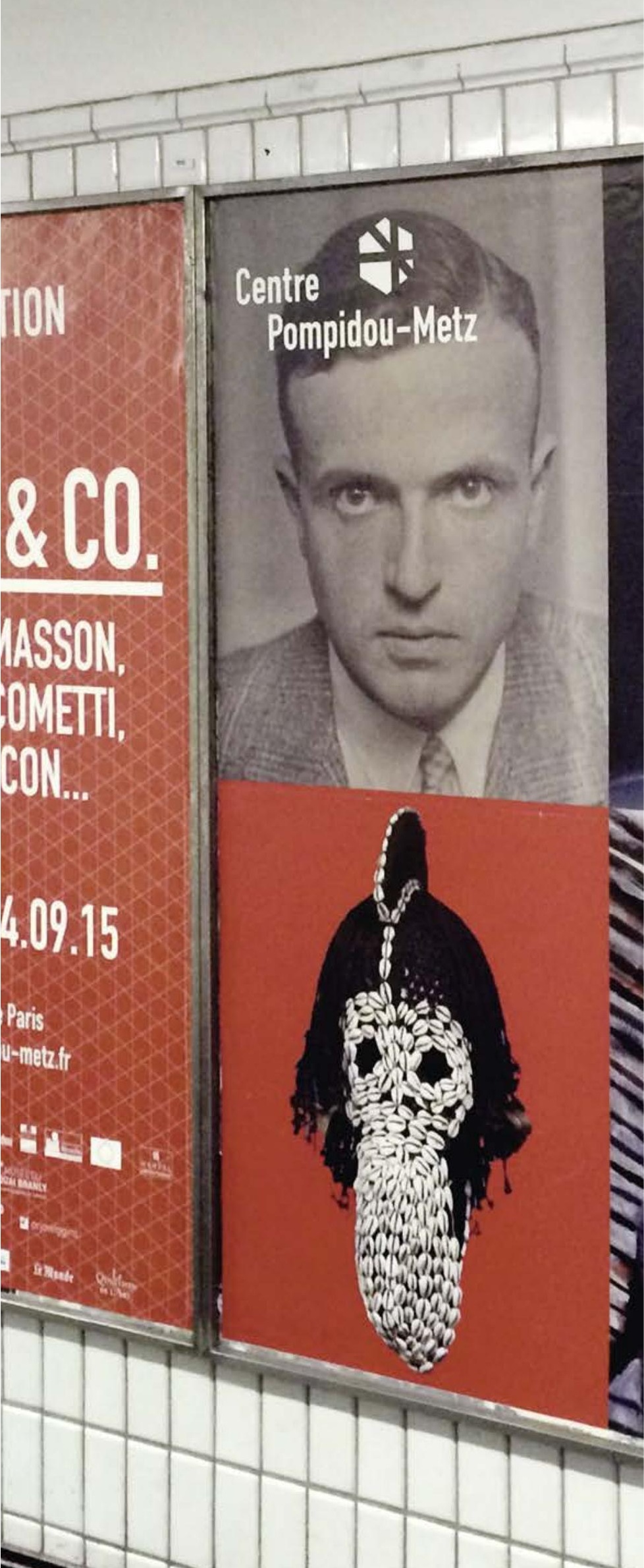


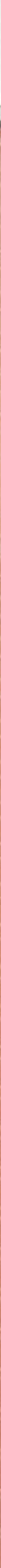

